\title{
EXPONENTIAL-SPLINE WAVELET BASES
}

\author{
Ildar Khalidov, Michael Unser
}

\author{
Biomedical Imaging Group, EPFL, Switzerland
}

\begin{abstract}
We build a multiresolution analysis based on shift-invariant exponential B-spline spaces. We construct the basis functions for these spaces and for their orthogonal complements. This yields a new family of wavelet-like basis functions of $L_{2}$, with some remarkable properties. The wavelets, which are characterized by a set of poles and zeros, have an explicit analytical form (exponential spline). They are nonstationary is the sense that they are scale-dependent and that they are not necessarily the dilates of one another. They behave like multi-scale versions of some underlying differential operator L; in particular, they are orthogonal to the exponentials that are in the null space of L. The corresponding wavelet transforms are implemented efficiently using an adaptation of Mallat's filterbank algorithm.
\end{abstract}

\section{INTRODUCTION}

During the past decade, the wavelet transform has become an essential tool in signal processing [1]. It is ideally suited for the processing of piecewise smooth signals because of the special way in which the basis functions (wavelets) interact with polynomials (vanishing moment property). The polynomial reproduction properties of the scaling function are also intimately linked to the crucial notion of approximation order. As the polynomials are carried only by the lowest part of the spectrum, the wavelet transform works best for signals whose energy is predominant at low frequencies. It is then natural to ask whether or not one can produce basis functions that are well suited to the representation of signals that are not necessarily lowpass; in particular, to those containing natural resonances. One solution, which is the one that we are developing here, is to substitute the polynomials of the classical wavelet theory by exponentials or even by sinusoids.

All the polynomial-related properties of wavelets have been traced back to the presence of the polynomial B-spline that lies hidden within [2]. If one transposes the argument to exponentials, one naturally hits upon the exponential Bsplines which, as their name may suggest, reconstruct exponentials perfectly [3]. These functions constitute the building blocks of our present wavelet design. They generate a generalized multiresolution analysis, in the sense defined by de Boor, DeVore, and Ron [4], that is non-stationary; in other words, it has all the usual properties, except that the basis functions at different scales are no longer dilates of each other.

The goal of the present work is to construct and characterize the wavelets that arise from the exponential B-spline multiresolution analysis (Sections 2 and 3), to study their properties (Section 4), to describe the fast decomposition/reconstruction algorithm (Section 5), and to present a few examples to illustrate their versatility (Section 6).

\section{E-SPLINE MULTIRESOLUTION}

Consider a linear differential system given by

$$
\begin{gathered}
\mathrm{D}^{N} y+a_{N-1} \mathrm{D}^{N-1} y+\cdots+a_{0} y= \\
\mathrm{D}^{M} x+b_{M-1} \mathrm{D}^{M-1} x+\cdots+b_{0} x,
\end{gathered}
$$

where $x(t)$ is the input signal, $y(t)$ is the system output, $M<N$. Let $\vec{\alpha}=\left\{\alpha_{n}\right\}_{n=1}^{N}$ and $\vec{\gamma}=\left\{\gamma_{m}\right\}_{m=1}^{M}$ be the roots of the polynomials $s^{N}+a_{N-1} s^{N-1}+\cdots+a_{0}$ and $s^{M}+b_{M-1} s^{M-1}+\cdots+b_{0}$, respectively. We denote $\mathrm{L}=$ $\mathrm{L}_{\vec{\alpha}, \vec{\gamma}}$ the corresponding operator, such that $\mathrm{L} y=x$. The Green function $\rho(t)=\rho_{\vec{\alpha}, \vec{\gamma}}(t)$ of the system is causal and satisfies $\mathrm{L} \rho=\delta$, where $\delta$ denotes the Dirac impulse; $\rho$ is given by

$$
\rho(t)=\mathcal{L}^{-1}\left\{\frac{\Pi_{m=1}^{M}\left(\cdot-\gamma_{m}\right)}{\Pi_{n=1}^{N}\left(\cdot-\alpha_{n}\right)}\right\}(t)
$$

and can be determined by explicitly computing the inverse Laplace transform. We call the spline-defining parameters, $\left\{\alpha_{n}\right\}_{n=1}^{N}$ and $\left\{\gamma_{m}\right\}_{m=1}^{M}$, the poles and the zeros, respectively.

A generalized E-spline with vector of poles $\vec{\alpha}$, vector of zeros $\vec{\gamma}$ and equally spaced knots $t_{k}=T k$ is a function that satisfies [3]

$$
\mathrm{L} s(t)=\sum_{k \in \mathbb{Z}} a[k] \delta(t-T k) .
$$

By applying the inverse operator $\mathrm{L}^{-1}$, we get

$$
s(t)=\sum_{k \in \mathbb{Z}} a[k] \rho(t-T k)+p(t),
$$


where $p(t)$ is a solution of the homogeneous equation $L\{p\}=$ 0 . In the case of uniform sampling, $p(t)$ can be reproduced

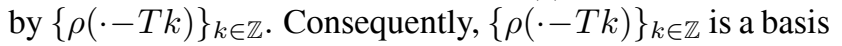
of the E-spline space.

An exponential B-spline $\beta_{T}(t)$ is defined by $\beta_{T}(t)=$ $\Delta_{\vec{\alpha}, T} \rho(t)$, where $\Delta_{\vec{\alpha}, T}=T\left(\Delta_{\left(\alpha_{1}\right), T} * \cdots * \Delta_{\left(\alpha_{n}\right), T}\right)$ is a localization operator with $\Delta_{\left(\alpha_{i}\right), T} f(t)=\frac{1}{T}\left(f(t)-e^{\alpha_{i} T} f(t-\right.$ $T)$ ). The localization process for the first-order case $(\vec{\alpha}=$ $\left.\left\{\alpha_{0}\right\}, \vec{\gamma}=\emptyset\right)$ is illustrated in Figure 1; the Green function, which is a causal exponential, is truncated by subtracting its weighted and shifted version.

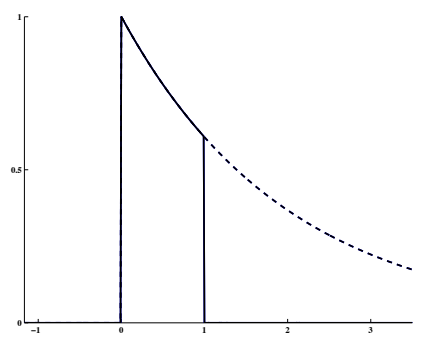

Fig. 1. Localization of the Green function for $T=1$.

The B-spline $\beta_{T}(t)$ is supported in $[0, T N)$. Its Fourier transform is given by

$$
\hat{\beta}_{T}(\omega)=\frac{1}{T^{N-1}} \prod_{k=1}^{N} \frac{1-e^{T(\alpha-j \omega)}}{\left(j \omega-\alpha_{k}\right)} \prod_{l=1}^{M}\left(j \omega-\gamma_{l}\right) .
$$

We assume the stability condition $\alpha_{l}-\alpha_{m} \neq 2 \pi k j / T$ for all distinct pure imaginary roots $\alpha_{l}, \alpha_{m}$. In this case, $\left\{\beta_{T}(\cdot-T k)\right\}_{k \in \mathbb{Z}}$ forms a Riesz basis [3, Theorem 1]. In addition, the Green function can be reconstructed as

$$
\rho(t)=\sum_{k=0}^{+\infty} p_{T}[k] \beta_{T}(t-T k),
$$

where $p_{T}[k]$ are some suitable weights [3]. Together with $\beta_{T} \in \operatorname{span}\{\rho(\cdot-T k)\}_{k \in \mathbb{Z}}$, this ensures that the exponential B-splines form a complete basis of the E-spline space.

We now consider the dyadic scales $T=2^{i}$ and define $V_{(i)}$ as

$$
V_{(i)}=\left\{s_{i}=\sum_{k \in \mathbb{Z}} c_{k} \rho\left(\cdot-2^{i} k\right)\right\} \cap L_{2},
$$

where the $c_{k}$ 's are arbitrary coefficients.

Due to the mentioned equivalence of bases, we can also write

$$
V_{(i)}=\left\{s_{i}(t)=\sum_{k \in \mathbb{Z}} c_{k} \varphi_{i}\left(t-2^{i} k\right): c \in l_{2}\right\},
$$

where $\varphi_{i}(t)=\beta_{2^{i}}(t) /\left\|\beta_{2^{i}}\right\|_{L_{2}}$ is the normalized scaling function.

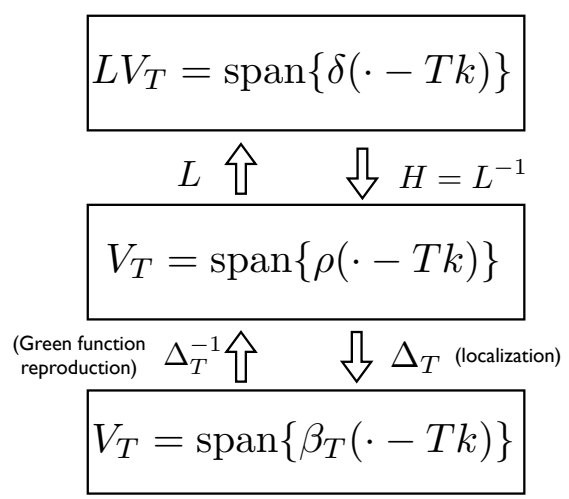

Fig. 2. E-spline space-construction process

We give in Figure 2 a diagram that summarizes the construction of the E-spline spaces.

The space $V_{(i)}$ is included in $V_{(i-1)}$ by construction. We therefore have a ladder of spaces

$$
\cdots \subset V_{(i+1)} \subset V_{(i)} \subset V_{(i-1)} \subset \ldots
$$

and we can prove that $\bigcup V_{(i)}$ is dense in $L_{2}$ [5].

\section{MULTIRESOLUTION BASIS FUNCTIONS}

The function $\varphi_{i+1} \in V_{(i+1)} \subset V_{(i)}$ can be decomposed as

$$
\varphi_{i+1}(t)=\sum_{k} h_{i}[k] \varphi_{i}\left(t-2^{i} k\right)
$$

which readily provides the explicit expression for the scaling filter

$$
H_{i}\left(e^{j 2^{i} \omega}\right)=\frac{\hat{\varphi}_{i+1}(\omega)}{\hat{\varphi}_{i}(\omega)}=2 \frac{c_{i}}{c_{i+1}} \cdot \prod_{k=1}^{N} \frac{1+e^{2^{i}\left(\alpha_{k}-j \omega\right)}}{2}
$$

where $c_{i}=\left\|\beta_{i}\right\|_{L_{2}}$ is a normalizing constant.

The dual scaling function is defined by

$$
\hat{\tilde{\varphi}}_{i}(\omega)=\hat{\varphi}_{i}(\omega) / A_{i}\left(e^{j 2^{i} \omega}\right)
$$

with $A_{i}\left(e^{j 2^{i} \omega}\right)=\sum_{k \in \mathbb{Z}} a_{i}[k] e^{-j 2^{i} \omega k}=2^{-i} \sum_{k \in \mathbb{Z}} \mid \hat{\varphi}_{i}(\omega+$ $\left.2 \pi k / 2^{i}\right)\left.\right|^{2}$, where $a_{i}[k]=\left\langle\varphi_{i}(\cdot), \varphi_{i}\left(\cdot-2^{i} k\right)\right\rangle$ is the Gramor autocorrelation-sequence of the basis $\left\{\varphi_{i}\left(t-2^{i} k\right)\right\}_{k \in \mathbb{Z}}$. $A_{i}(z)$, the $z$-transform of $a_{i}[k]$, is also referred to as the autocorrelation filter.

It can be proved that $\left\{\tilde{\varphi}_{i}\left(\cdot-2^{i} k\right)\right\}$ is a Riesz basis of $V_{(i)}$ [5]. The dual scaling filter is then obtained as

$$
\tilde{H}_{i}(z)=\frac{A_{i}(z)}{A_{i+1}\left(z^{2}\right)} H_{i}(z)
$$


and the dual two-scale relation is

$$
\tilde{\varphi}_{i+1}(t)=\sum_{k} \tilde{h}_{i}[k] \tilde{\varphi}_{i}\left(t-2^{i} k\right) .
$$

The wavelet function

$$
\psi_{i+1}(t)=\sum_{k} g_{i}[k] \varphi_{i}\left(t-2^{i} k\right)
$$

is obtained by solving for $g_{i}[k]$ the orthogonality relation

$$
\left\langle\psi_{i+1}(\cdot), \varphi_{i+1}\left(\cdot-2^{i+1} k\right)\right\rangle=0
$$

The general solution is given by

$$
G_{i}(z)=-z Q_{i}\left(z^{2}\right) H_{i}^{*}\left(-z^{-1}\right) A_{i}(-z),
$$

where the filter $Q_{i}(z)$ must be chosen to be bounded and non-vanishing on the unit circle [5]. By imposing the perfectreconstruction condition, we get as well the dual scaling filter

$$
\tilde{G}_{i}(z)=-z \frac{\tilde{H}_{i}^{*}\left(-z^{-1}\right)}{A_{i}(-z) Q_{i}^{*}\left(z^{-2}\right)},
$$

where $\tilde{g}_{i}[k]$ are the coefficients in the dual wavelet relation

$$
\tilde{\psi}_{i+1}(t)=\sum_{k} \tilde{g}_{i}[k] \tilde{\varphi}_{i}\left(t-2^{i} k\right)
$$

The constructed scaling functions and wavelets at each scale $i$ belong to the space $C^{N-M-2}$. The scaling and the wavelet filters are either FIR (B-spline case) or IIR with exponential decay (dual case). Thus, the basis functions are either compactly supported, or exponentially decaying. They can also be orthogonalized to yield orthonormal wavelets.

\section{PROPERTIES}

The new wavelet bases have the following remarkable properties, which may be useful in signal-processing applications:

Property 1 (Reproduction of exponential polynomials). Suppose that $\vec{\alpha}$ contains a root $\alpha_{0}$ of multiplicity $m$. Then, for $n=0, \ldots, m-1$ the exponential monomial $t^{n} e^{\alpha_{0} t}$ has the $B$-spline representation

$$
t^{n} e^{\alpha_{0} t}=\sum_{k \in \mathbb{Z}} p_{\vec{\alpha}, i, n}[k] \beta_{\vec{\alpha}, \vec{\gamma}, 2^{i}}\left(t-2^{i} k\right),
$$

where $p_{\vec{\alpha}, i, n}[k]$ are suitable coefficients.

For the arbitrary parameter vector $\vec{\alpha}$ consisting of $N_{d}$ distinct roots of multiplicity $m_{k}, k=1, \ldots, N_{d}$, the direct corollary is that the exponential polynomials

$$
p_{\vec{\alpha}}(t)=\sum_{k=1}^{N_{d}} \sum_{n=0}^{m_{k}-1} c_{k} t^{n} e^{\alpha_{k} t}
$$

that constitute the null space $N_{\vec{\alpha}}$ of the operator L can be reproduced with the corresponding B-splines.
Property 2 (Vanishing exponential moments). For each scale $i \in \mathbb{Z}$, shift $t_{0} \in \mathbb{R}$, and degree $n=0, \ldots, m_{k}-1$, the analysis wavelet satisfies

$$
\int_{-\infty}^{\infty} t^{n} e^{\alpha_{k} t} \psi_{i}\left(t-t_{0}\right) \mathrm{d} t=0
$$

where $m_{k}$ is the multiplicity of $\alpha_{k}$. In other words, the analysis wavelet $\psi_{i}$ has $N$ vanishing exponential moments that correspond to the basis functions of the null space of $\mathrm{L}$.

Property 3 (Operator-like wavelets). Let $\left\{\psi_{i, k}\right\}_{i, k \in \mathbb{Z}}$ be an E-spline wavelet basis of $L_{2}$. Then, there exists a se-

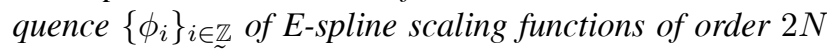
such that $\left\langle f, \tilde{\psi}_{i}\left(\cdot-t_{0}\right)\right\rangle=\mathrm{L}\left\{f * \phi_{i}\right\}\left(t_{0}\right)$; in addition, $\left\{\phi_{i}\right\}_{i \in \mathbb{Z}}$ generates a multiresolution analysis of $L_{2}$. The wavelet coefficients of $f$ are therefore the samples of the smoothed versions of $L_{\vec{\alpha}, \vec{\gamma}} f$.

\section{IMPLEMENTATION}

To perform the decomposition and the reconstruction in the new bases, we use an adapted version of the fast Mallat's filterbank algorithm with scale-dependent filters. The filters $G_{i}(z), H_{i}(z), \tilde{G}_{i}(z), \tilde{H}_{i}(z)$ should be pre-calculated at each scale according to the formulas presented in Section 3.

In order to initialize the decomposition, we need to obtain the initial projection coefficients $c[k]$ such that $f\left(k_{0}\right)=$ $\sum_{k \in \mathbb{Z}} c[k] \varphi_{0}\left(k_{0}-k\right)$, as well as the autocorrelation filter $A_{0}(z)$. The $c[k]$ 's can be obtained by prefiltering the function samples with $P(z)=\left(\sum_{k} \varphi_{0}(k) z^{-k}\right)^{-1}$. Both $P(z)$ and $A_{0}(z)$ can be calculated by applying fractional differences to the samples of the Green function $\rho$. The explicit formula for $\rho(t)$ is obtained by performing a partial-fraction decomposition in the Laplace domain [5].

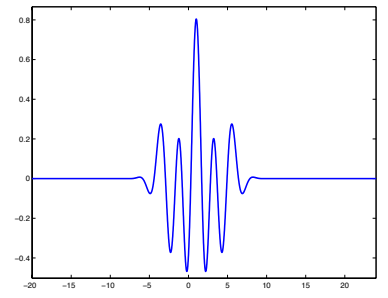

(a) Wavelet $\psi_{1}, i=1$

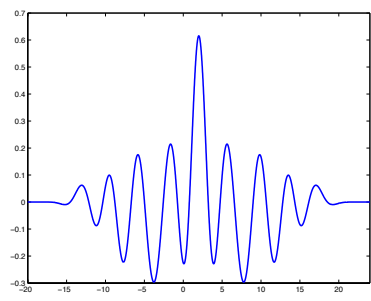

(b) Wavelet $\psi_{2}, i=2$
Fig. 3. Wavelets at scales $i=1, i=2 ; \vec{\alpha}=$ $\left(-\frac{5 \pi}{8} j,-\frac{5 \pi}{8} j, \frac{5 \pi}{8} j, \frac{5 \pi}{8} j, 0,0\right), \vec{\gamma}=(-5 j, 5 j)$.

\section{EXAMPLES}

To illustrate the variety of time-domain and spectral shapes that can be achieved with this construction, we choose the 
parameters $\vec{\alpha}=\left(-\frac{5 \pi}{8} j,-\frac{5 \pi}{8} j, \frac{5 \pi}{8} j, \frac{5 \pi}{8} j, 0,0\right)$ and $\vec{\gamma}=$ $(-5 j, 5 j)$ to be pure imaginary and to have Hermitian symmetry, which ensures that the time-domain functions are real. In Figure 3, we show the wavelets $\psi_{1}$ and $\psi_{2}$ in the time domain; these have a strong oscillatory character, with a larger number of lobes as the scale gets coarser. It is clearly apparent that $\psi_{2}$ is not a dilate of $\psi_{1}$.

The plots in Figure 4 show the effect of the poles $\vec{\alpha}$ and of the zeros $\vec{\gamma}$ on the frequency response. In Figure 4(a), the

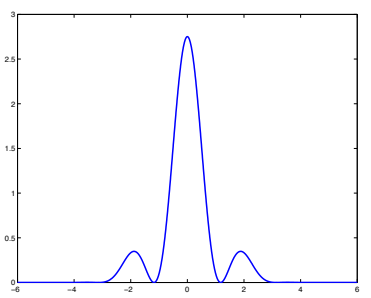

(a) Scaling-function spectrum $\left|\hat{\varphi}_{1}(\omega)\right|, i=1$

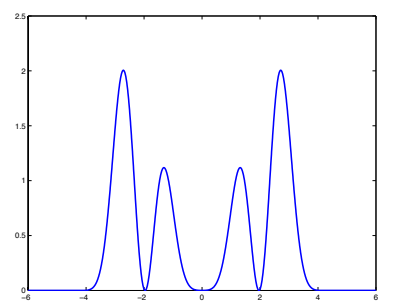

(b) Wavelet spectrum $\left|\hat{\psi}_{1}(\omega)\right|, i=1$
Fig. 4. The spectrum of the scaling function and the spectrum of the wavelet; $\vec{\alpha}=\left(-\frac{5 \pi}{8} j,-\frac{5 \pi}{8} j, \frac{5 \pi}{8} j, \frac{5 \pi}{8} j, 0,0\right)$, $\vec{\gamma}=(-5 j, 5 j)$.

pole $\alpha_{1}=-\frac{5 \pi}{8} j$ produces a peak of the scaling-function spectrum near $\omega=-\frac{5 \pi}{8} j$ and makes it vanish with periodicity $\pi$. There is also a peak at the origin because of the pole at $\omega=0$. The zero $\gamma_{1}=-5 j$ makes the frequency response vanish at $\omega=-5$. In contrast, the wavelet spectrum (Figure 4(b)) vanishes at $j \omega=\alpha_{l}$, as a result of the vanishing exponential moment and of the linear-differential-operator properties of E-spline wavelets.

\section{CONCLUSION}

We have introduced a general procedure for constructing new exponential-spline wavelet-like bases of $L_{2}$. Similar to analog filters, the multiresolution spaces are characterized by a set of poles and zeros. The wavelets come in different flavors: basic (B-spline), dual, and orthonormal. They have the ability to kill exponential polynomials, which generalizes the notion of vanishing moment found in the conventional wavelet theory. More importantly, they essentially behave like multiscale versions of some underlying operator $L$. One recovers the polynomial B-spline case by choosing the parameter $\vec{\alpha}=(0, \ldots, 0)$, where the corresponding operator is the $N$-th derivative.

The constructed wavelets are not dilates of a single function anymore; however, they still can be implemented using a scale-dependent version of Mallat's fast filterbank algorithm.
The proposed framework should be of interest for signalprocessing applications. It might be well-suited for representing signals that are not predominantly lowpass but that have substantial energy in other frequency bands. In particular, it offers the possibility of adapting the model to a given class of signals, by the way of selecting roots that fit natural resonances in the data.

\section{ACKNOWLEDGEMENTS}

This work is funded in part by grant 200020-101821 from the Swiss National Science Foundation.

\section{REFERENCES}

[1] S. Mallat, A Wavelet Tour of Signal Processing, Academic Press, San Diego, 1998.

[2] M. Unser and T. Blu, "Wavelet theory demystified," IEEE Trans. Signal Process., vol. 51, no. 2, pp. 470483, February 2003.

[3] M. Unser, "Cardinal exponential splines: Part IIThink analog, act digital," IEEE Trans. Signal Processing, in press.

[4] C. de Boor, R.A. DeVore, and A. Ron, "On the construction of multivariate (pre)wavelets," Constr. Approx., vol. 2, no. 3, pp. 123-166, 1993.

[5] I. Khalidov and M. Unser, "From differential equations to the construction of new wavelet-like bases," IEEE Trans. Signal Processing, submitted. 